\title{
Roberge-Weiss transitions at different center symmetry breaking patterns in a $\mathbb{Z}_{3}$-QCD model
}

\author{
Xiu-Fei Liø and Zhao Zhang $\odot^{*}$ \\ School of Mathematics and Physics, North China Electric Power University, Beijing 102206, China
}

(Received 15 August 2019; published 24 October 2019)

\begin{abstract}
We study how the Roberge-Weiss (RW) transition depends on the pattern of center symmetry breaking using a $\mathbb{Z}_{3}$-QCD model. We adopt flavor-dependent quark imaginary chemical potentials, namely, $\left(\mu_{u}, \mu_{d}, \mu_{s}\right) / i T=(\theta-2 \pi C / 3, \theta, \theta+2 \pi C / 3)$ with $C \in[0,1]$. The RW periodicity is guaranteed and the center symmetry of $\mathbb{Z}_{3}$-QCD is explicitly broken when $C \neq 1$ and/or quark masses are nondegenerate. For $N_{f}=3$ and $C \neq 1$, the RW transition occurs at $\theta=\theta_{\mathrm{RW}}=(2 k+1) \pi / 3(k \in \mathbb{Z})$, which becomes stronger with decrease of $C$. When $C=1$, the $\theta_{\mathrm{RW}}$ turns into $2 k \pi / 3$ for $N_{f}=2+1$, but keeps $(2 k+1) \pi / 3$ for $N_{f}=1+2$; in both cases, the RW transitions get stronger with the mass mismatch. For other $C \neq 0$ cases, the $\theta_{\mathrm{RW}}$ 's are not integral multiples of $\pi / 3$. We find that the RW transition is more sensitive to the deviation of $C$ from one compared to the mass nondegeneracy, and thus the strength of the traditional RW transition with $C=0$ is the strongest. The nature of RW end points and its implications to deconfinement transition are investigated.
\end{abstract}

DOI: 10.1103/PhysRevD.100.074026

\section{INTRODUCTION}

Exploration of the quantum chromodynamics (QCD) phase diagram at finite temperature and density is one of the most challenging subjects in particle and nuclear physics. As a first-principle method, the lattice QCD (LQCD) simulations yield many meaningful results at vanishing baryon chemical potential (see [1] and references therein). However, it is still unavailable in the nonzero real chemical potential region because of the well-known sign problem [2]. To evade this difficulty, various methods have been developed [3-8]. One useful approach is the analytic continuation from imaginary to real chemical potential [7-9], in which the fermion determinant is real and thus free from the sign problem.

Introducing an imaginary chemical potential $\mu_{I}=i \theta T$ in QCD corresponds to replacing the fermion antiperiodic boundary condition $(\mathrm{ABC})$ by the twisted one. In this case, the partition function satisfies $Z_{\mathrm{QCD}}(\theta)=Z_{\mathrm{QCD}}(\theta+2 \pi / 3)$, which is called the Roberge-Weiss (RW) periodicity [10]. Since the $\mathbb{Z}_{3}$ symmetry is broken by dynamical quarks, the effective thermal potentials $\Omega_{\phi}(\phi=0, \pm 2 \pi / 3)$ of three $\mathbb{Z}_{3}$ sectors differ from each other above some critical temperature $T_{\mathrm{RW}}$ and the physical solution is determined by the

\footnotetext{
*zhaozhang@pku.org.cn
}

Published by the American Physical Society under the terms of the Creative Commons Attribution 4.0 International license. Further distribution of this work must maintain attribution to the author(s) and the published article's title, journal citation, and DOI. Funded by SCOAP ${ }^{3}$. absolute minimum of the three ones. This leads to a discontinuity of $d \Omega_{\mathrm{QCD}}(\theta) / d \theta$ at $\theta=\pi / 3 \bmod 2 \pi / 3$, which is known as the RW transition [10].

The RW transition is a true phase transition for the $\mathbb{Z}_{2}$ symmetry. LQCD simulations suggest that the nature of the RW end point may depend on quark masses [11-19]: For intermediate quark masses, it is a critical end point, while for large and small quark masses it is a triple point. The latest LQCD calculation provides evidence that the RW end point transition remains second order, in the 3D Ising universality class, in the explored mass range corresponding to $m_{\pi} \simeq 100,70$, and $50 \mathrm{MeV}$ [20]. The RW transition has also been investigated in effective models of QCD [21-26]. Due to the analogy between $\theta$ and the AharonovBohm phase, it is proposed that the RW transition can be considered as a topological phase transition [27].

Note that special flavor-twisted boundary conditions (FTBCs) can lead to an unbroken $\mathbb{Z}_{N_{c}}$ center symmetry. As shown in [28,29], for $N_{f}$ flavors with a common mass in the fundamental representation, the $S U\left(N_{c}\right)$ gauge theory with $d \equiv \operatorname{gcd}\left(N_{f}, N_{c}\right)>1$ has a $\mathbb{Z}_{d}$ color-flavor center symmetry when imposing the $\mathbb{Z}_{N_{f}}$-symmetric FTBCs on $S^{1}$. The $\mathbb{Z}_{d}$ symmetry arises due to the intertwined color center transformations and cyclic flavor permutations. The QCD-like theory for $N_{c}=N_{f}=3$ under such FTBCs is termed as $\mathbb{Z}_{3}$-QCD [28]. In this theory, the Polyakov loop is the true order parameter for center symmetry (even fermions appear). $\mathbb{Z}_{3}-\mathrm{QCD}$ is an interesting and instructive theory that is useful for understanding the deconfinement transition of QCD [28-35]. 
As mentioned, FTBCs on $S^{1}$ in $\mathbb{Z}_{3}$-QCD can be replaced with the standard fermion ABCs by introducing $\mu_{f}=i \theta_{f} T$ (shifted by $i 2 \pi T / 3$ ). Correspondingly, the center symmetry of $\mathbb{Z}_{3}$-QCD can be explicitly broken by mass nondegeneracy of quarks, or no equal $2 \pi / 3$ difference in $\theta_{f}$, or both if color and flavor numbers are unchanged. Then, some natural and interesting questions arise: Can the $\mathbb{Z}_{3}$ symmetry breaking in such ways lead to RW transitions? How do these RW transitions depend on the center symmetry breaking? What are the differences between these RW transitions and the traditional ones in QCD with a flavorindependent $\mu_{I}$ ? Answering these questions may deepen our understanding of the relationship between $\mathbb{Z}_{3}$ symmetry, RW transition, and deconfinement transition. Actually, one advantage of $\mathbb{Z}_{3}$-QCD is that we can use it to study how the pattern and degree of $\mathbb{Z}_{3}$ symmetry breaking can affect the nature of RW and deconfinement transitions from a different perspective.

The main purpose of this work is to study how RW transitions depend on the center symmetry breaking patterns by using a $\mathbb{Z}_{3}$-QCD model. We employ the three-flavor Polyakov-loop-extended Nambu Jona-Lasinio (PNJL) model [36,37], which possesses the so-called extended $\mathbb{Z}_{3}$ symmetry and can correctly reproduce the RW periodicity [38]. Without loss of generality, the flavordependent imaginary chemical potentials $\left(\mu_{u}, \mu_{d}, \mu_{s}\right) / i T=$ $(\theta-2 \pi C / 3, \theta, \theta+2 \pi C / 3)$ with $0 \leq C \leq 1$ are adopted, which guarantees $Z(\theta)=Z(\theta+2 \pi / 3)$ [26]. When quark masses are nondegenerate or $C \neq 1$, the center symmetry of $\mathbb{Z}_{3}$-QCD is explicitly broken and the RW transitions should appear at high temperature. We focus on five types of center symmetry breaking and study impacts of variations of $C$ and quark masses on the RW and deconfinement transitions.

The paper is organized as follows. In Sec. II, $\mathbb{Z}_{3}-\mathrm{QCD}$ and the PNJL model with flavor-dependent imaginary chemical potentials are introduced. In Sec. III, we present the results of the numerical calculation. Section IV gives the discussion and conclusion.

\section{II. $\mathbb{Z}_{3}$-QCD AND $\mathbb{Z}_{3}$-SYMMETRIC PNJL MODEL}

\section{A. $\mathbb{Z}_{3}-\mathbf{Q C D}$}

The $\mathbb{Z}_{3}$ transformation of QCD is defined as

$q \rightarrow q^{\prime}=U q, \quad A_{\mu} \rightarrow A_{\mu}{ }^{\prime}=U A_{\mu} U^{-1}+i\left(\partial_{\mu} U\right) U^{-1}$,

where $U$ is an element of the $S U(3)$ group, which satisfies the temporal boundary condition

$$
U\left(x_{4}=\beta, \mathbf{x}\right)=z_{k} U\left(x_{4}=0, \mathbf{x}\right),
$$

with $z_{k}=e^{-i 2 \pi k / 3}$ being an element of the center group. Even the QCD partition function $Z_{\mathrm{QCD}}$ is invariant under the $\mathbb{Z}_{3}$ transformation, and the original quark $\mathrm{ABC}$

$$
q\left(x_{4}=\beta, \mathbf{x}\right)=-q\left(x_{4}=0, \mathbf{x}\right)
$$

is changed into

$$
q\left(x_{4}=\beta, \mathbf{x}\right)=-e^{i 2 \pi k / 3} q\left(x_{4}=0, \mathbf{x}\right) .
$$

Thus, the center symmetry is explicitly broken due to (4). This is why the Polyakov loop is no longer the true order parameter of deconfinement in QCD.

However, the $\mathbb{Z}_{3}$ symmetry can be recovered if one consider the FTBCs [28]

$$
q_{f}\left(x_{4}=\beta, \mathbf{x}\right)=-e^{-i \theta_{f}} q_{f}\left(x_{4}=0, \mathbf{x}\right),
$$

with

$$
\theta_{f}=\frac{2 \pi}{3} f \quad(f=-1,0,1)
$$

instead of the ABCs. For convenience, three numbers $-1,0$, and 1 are used as the flavor indices. Under the $\mathbb{Z}_{3}$ transformation, the FTBCs are transformed into

$$
q_{f}\left(x_{4}=\beta, \mathbf{x}\right)=-e^{-i \theta_{f}^{\prime}} q_{f}\left(x_{4}=0, \mathbf{x}\right),
$$

with

$$
\theta_{f}^{\prime}=\frac{2 \pi}{3}(f-k) \quad(f=-1,0,1)
$$

We can see that the modified boundary conditions (7) return to the original ones (5) if the flavor indices $f-k$ are relabeled as $f$. This means the QCD-like theory with the FTBCs (5) is invariant under the center transformation if three flavors have a common mass. As mentioned, such a theory is termed as $\mathbb{Z}_{3}-\mathrm{QCD}$, which equals QCD when $T \rightarrow 0$.

The FTBCs (5) can be changed back into the standard ABCs through the field transformation [10]

$$
q_{f} \rightarrow e^{-i \theta_{f} T x_{4}} q_{f},
$$

which gives rise to the flavor-dependent imaginary chemical potentials

$$
\mu_{f}=i \theta_{f} T
$$

This implies that the global $S U_{V}(3) \otimes S U_{A}(3)$ symmetry in the chiral limit is broken to $\left(U_{V}(1)\right)^{2} \otimes\left(U_{A}(1)\right)^{2}$ in $\mathbb{Z}_{3}$-QCD [32].

Equation (4) and the transformation between FTBCs (5) and imaginary chemical potentials (10) indicate the RW periodicity: the partition function $Z\left(\theta_{f}\right)$ is periodic under the shifts 


$$
\mu_{f} / i T \rightarrow \mu_{f} / i T+2 \pi / 3
$$

i.e.,

$$
Z\left(\theta_{f}\right)=Z\left(\theta_{f}+2 \pi / 3\right) .
$$

\section{B. $\mathbb{Z}_{3}$-symmetry breaking patterns in $\mathbb{Z}_{3}$-QCD}

The center symmetry of $\mathbb{Z}_{3}$-QCD is attributed to three conditions: (1) $N_{f}=N_{c}=3$ [namely, $\operatorname{gcd}\left(N_{f}, N_{c}\right)>1$ ], (2) quark masses are degenerate, and (3) the dimensionless flavor-dependent imaginary chemical potentials (normalized by $i T$ ) form an arithmetic sequence with the common difference $2 \pi / N_{c}$. Correspondingly, the center symmetry of $\mathbb{Z}_{3}$-QCD will be broken explicitly if any one of these conditions is not satisfied, which may lead to the RW transition at high temperature. It is interesting to study how the possible RW transitions depend on the changes of conditions 2 and/or 3 in $\mathbb{Z}_{3}$-QCD by keeping $N_{f}=N_{c}=3$.

Here we express the imaginary chemical potential matrix $\hat{\mu}=\operatorname{diag}\left(\mu_{u}, \mu_{d}, \mu_{s}\right)=i T \hat{\theta}$ in terms of two real parameters $\theta$ and $C$, namely,

$$
\hat{\theta}=\left(\begin{array}{lll}
\theta_{u} & & \\
& \theta_{d} & \\
& & \theta_{s}
\end{array}\right)=\left(\begin{array}{lll}
\theta-\frac{2 \pi C}{3} & & \\
& \theta & \\
& & \theta+\frac{2 \pi C}{3}
\end{array}\right),
$$

where $C \in[0,1]$. As mentioned, such a choice of $\hat{\theta}$ ensures the RW periodicity $Z(\theta)=Z(\theta+2 \pi / 3)$. We concentrate on the following center symmetry breaking patterns: (i) $N_{f}=3$ with varied $C \neq 1$ (here and after $N_{f}=3$ means three flavors share the same mass), (ii) $N_{f}=2+1$ (two lighter flavors have the same mass) with $C=1$, (iii) $N_{f}=1+2$ (two heavier flavors have the same mass) with $C=1$, (iv) $N_{f}=2+1$ with varied $C \neq 1$, and (v) $N_{f}=1+1+1$ with $C=1$.

For cases (i)-(iii), the thermal dynamical potential $\Omega(\theta)$ is a $\theta$-even function even if the imaginary chemical potentials are flavor dependent. For case (i), three flavors are mass degenerate and thus

$$
\begin{aligned}
\Omega(\theta) & =\Omega\left(\theta_{u}, \theta_{d}, \theta_{s}\right) \\
& =\Omega(\theta-2 \pi C / 3, \theta, \theta+2 \pi C / 3) \\
& \stackrel{\mathcal{C}}{\rightarrow} \Omega(-\theta+2 \pi C / 3,-\theta,-\theta-2 \pi C / 3) \\
& \stackrel{u \leftrightarrow s}{=} \Omega(-\theta-2 \pi C / 3,-\theta,-\theta+2 \pi C / 3) \\
& =\Omega(-\theta),
\end{aligned}
$$

where $\stackrel{\mathcal{C}}{\rightarrow}$ stands for the charge conjugation transformation and $\Omega(\hat{\theta})=\Omega(-\hat{\theta})$ always holds. For cases (ii) and (iii), two flavors are mass degenerate (e.g., $m_{u}=m_{d}$ ) and thus

$$
\begin{aligned}
\Omega(\theta) & =\Omega\left(\theta_{u}, \theta_{d}, \theta_{s}\right) \\
& =\Omega(\theta-2 \pi / 3, \theta, \theta+2 \pi / 3) \\
& \stackrel{\mathcal{C}}{\rightarrow} \Omega(-\theta+2 \pi / 3,-\theta,-\theta-2 \pi / 3) \\
& \underline{\underline{ } \rightarrow \theta+4 \pi / 3} \Omega(-\theta-2 \pi / 3,-\theta-4 \pi / 3,-\theta) \\
& \underline{\underline{u \leftrightarrow d}} \Omega(-\theta-4 \pi / 3,-\theta-2 \pi / 3,-\theta) \\
& \underline{\underline{\theta \rightarrow \theta-2 \pi / 3}} \Omega(-\theta-2 \pi / 3,-\theta,-\theta+2 \pi / 3) \\
& =\Omega(-\theta) .
\end{aligned}
$$

Note that $\Omega(\theta)=\Omega(-\theta)$ does not hold for cases (iv) and (v).

\section{The center-symmetric PNJL model}

The Lagrangian of the three-flavor PNJL model of QCD in Euclidean spacetime is defined as [36,37]

$$
\begin{aligned}
\mathcal{L}= & \bar{q}\left(\gamma_{\mu} D_{\mu}+\hat{m}-\hat{\mu} \gamma_{4}\right) q-G_{\mathrm{S}} \sum_{a=0}^{8}\left[\left(\bar{q} \lambda_{f}^{a} q\right)^{2}+\left(\bar{q} i \gamma_{5} \lambda_{f}^{a} q\right)^{2}\right] \\
& +G_{\mathrm{D}}\left[\operatorname{det}_{i j} \bar{q}_{i}\left(1+\gamma^{5}\right) q_{j}+\operatorname{det}_{i j} \bar{q}_{i}\left(1-\gamma^{5}\right) q_{j}\right] \\
& +\mathcal{U}\left(\Phi[A], \Phi^{*}[A], T\right),
\end{aligned}
$$

where $D_{\mu}=\partial_{\mu}+i g_{s} \delta_{\mu 4} A_{\mu}^{a} \lambda^{a} / 2$ is the covariant derivative with the $S U(3)$ gauge coupling $g_{s}$ and Gell-Mann matrices $\lambda^{a}, \hat{m}=\operatorname{diag}\left(m_{u}, m_{d}, m_{s}\right)$ denotes the current quark mass matrix, and $\hat{\mu}=\operatorname{diag}\left(\mu_{u}, \mu_{d}, \mu_{s}\right)$ is the quark chemical potential matrix. $G_{\mathrm{S}}$ and $G_{\mathrm{D}}$ are the coupling constants of the scalar-type four-quark interaction and the KobayashiMaskawa-'t Hooft determinant interaction [39-41], respectively.

The Polyakov-loop potential $\mathcal{U}\left(\Phi[A], \Phi^{*}[A], T\right)$ in the Lagrangian (16) is center symmetric, which is the function of the Polyakov loop $\Phi$ and its conjugate $\Phi^{*}$ and $T$. The quantity $\Phi$ is the true order parameter for center symmetry in pure gauge theory (and also in $\mathbb{Z}_{3}$-QCD), which is defined as

$$
\Phi=\frac{1}{3} \operatorname{Tr}(L),
$$

with

$$
L(\mathbf{x})=\mathcal{P} \exp \left[i \int_{0}^{\beta} d \tau A_{4}(\mathbf{x}, \tau)\right],
$$

where $\mathcal{P}$ is the path-integral ordering operator. One popular Polyakov-loop potential is the logarithmic one proposed in [42], which takes the form

$$
\begin{aligned}
\mathcal{U}\left(\Phi, \Phi^{*}, T\right)= & T^{4}\left[-\frac{a(T)}{2} \Phi \Phi^{*}+b(T) \ln \left(1-6 \Phi \Phi^{*}\right.\right. \\
& \left.\left.+4\left(\Phi^{3}+\Phi^{* 3}\right)-3\left(\Phi \Phi^{*}\right)^{2}\right)\right]
\end{aligned}
$$


where

$$
a(T)=a_{0}+a_{1}\left(\frac{T_{0}}{T}\right)+a_{2}\left(\frac{T_{0}}{T}\right)^{2}, \quad b(T)=b_{3}\left(\frac{T_{0}}{T}\right)^{3} .
$$

The potential (19) will be used in our calculation.

In the Polyakov gauge, the matrix $L$ can be represented as a diagonal form in the color space

$$
L=\mathrm{e}^{i \beta A_{4}}=\operatorname{diag}\left(\mathrm{e}^{i \beta \phi_{1}}, \mathrm{e}^{i \beta \phi_{2}}, \mathrm{e}^{i \beta \phi_{3}}\right),
$$

where $\phi_{1}+\phi_{2}+\phi_{3}=0$. The mean-field thermodynamic potential of PNJL then reads

$$
\begin{aligned}
\Omega= & 2 G_{\mathrm{S}} \sum_{f} \sigma_{f}^{2}-4 G_{\mathrm{D}} \sigma_{u} \sigma_{d} \sigma_{s}-\frac{2}{\beta} \sum_{f} \int \frac{d^{3} \mathbf{p}}{(2 \pi)^{3}}\left[3 \beta E_{f}\right. \\
& +\ln \left(1+3 \Phi \mathrm{e}^{-\beta\left(E_{f}-\mu_{f}\right)}+3 \Phi^{*} \mathrm{e}^{-2 \beta\left(E_{f}-\mu_{f}\right)}+\mathrm{e}^{-3 \beta\left(E_{f}-\mu_{f}\right)}\right) \\
& \left.+\ln \left(1+3 \Phi^{*} \mathrm{e}^{-\beta\left(E_{f}+\mu_{f}\right)}+3 \Phi \mathrm{e}^{-2 \beta\left(E_{f}+\mu_{f}\right)}+\mathrm{e}^{-3 \beta\left(E_{f}+\mu_{f}\right)}\right)\right] \\
& +\mathcal{U},
\end{aligned}
$$

with $\sigma_{f}=\left\langle\bar{q}_{f} q_{f}\right\rangle$ and $E_{f}=\sqrt{\mathbf{p}^{2}+M_{f}^{2}}(f=u, d, s)$. The dynamical quark masses are defined by

$$
M_{f}=m_{f}-4 G_{\mathrm{S}} \sigma_{f}+2 G_{\mathrm{D}} \sigma_{f^{\prime}} \sigma_{f^{\prime \prime}},
$$

where $f \neq f^{\prime}, f^{\prime} \neq f^{\prime \prime}$, and $f \neq f^{\prime \prime}$. As usual, the threedimensional cutoff $\Lambda$ is introduced to regularize the vacuum contribution. For the pure imaginary chemical potential case, we can write $\Phi$ and $\Phi^{*}$ as

$$
\Phi=R e^{i \phi}, \quad \Phi^{*}=R e^{-i \phi} .
$$

The condensates $\sigma_{f}$, the magnitude $R$, and the phase $\phi$ are determined by the stationary conditions

$$
\frac{\partial \boldsymbol{\Omega}}{\partial \sigma_{u}}=\frac{\partial \boldsymbol{\Omega}}{\partial \sigma_{d}}=\frac{\partial \Omega}{\partial \sigma_{s}}=\frac{\partial \boldsymbol{\Omega}}{\partial R}=\frac{\partial \boldsymbol{\Omega}}{\partial \phi}=0 .
$$

Similar to $\mathbb{Z}_{3}-\mathrm{QCD}$, the three-flavor PNJL with a common quark mass possesses the exact $\mathbb{Z}_{3}$ symmetry when introducing the special flavor-dependent imaginary chemical potentials $\hat{\mu}=i T \hat{\theta}$, where $\hat{\theta}=\operatorname{diag}(\theta-2 \pi / 3, \theta$, $\theta+2 \pi / 3)$ [28]. Here we refer to this center-symmetric PNJL as $\mathbb{Z}_{3}$-PNJL, which can be regarded as a low-energy effective theory of $\mathbb{Z}_{3}-\mathrm{QCD}$. The RW transitions under conditions (i)-(v) will be studied in the $\mathbb{Z}_{3}$-PNJL formalism by breaking the center symmetry explicitly.

\section{Model parameters}

The five parameters of the logarithmic Polyakov-loop potential (19) are listed in Table I. Originally, $T_{0}$ is the
TABLE I. The parameter set of the Polyakov-loop potential.

\begin{tabular}{lcccc}
\hline \hline$a_{0}$ & $a_{1}$ & $a_{2}$ & $b_{3}$ & $T_{0}(\mathrm{MeV})$ \\
\hline 3.51 & -2.47 & 15.2 & -1.75 & 195 \\
\hline \hline
\end{tabular}

TABLE II. The parameter set in the NJL sector.

\begin{tabular}{ccccc}
\hline \hline$m_{u(d)}(\mathrm{MeV})$ & $m_{s}(\mathrm{MeV})$ & $\Lambda(\mathrm{MeV})$ & $G_{\mathrm{S}} \Lambda^{2}$ & $G_{D} \Lambda^{5}$ \\
\hline 5.5 & 140.7 & 602.3 & 1.835 & 12.36 \\
\hline \hline
\end{tabular}

critical temperature of deconfinement for pure $S U(3)$ gauge theory, which is around $270 \mathrm{MeV}$ [43,44]. Note that the chiral $T_{c}$ at zero density obtained in PNJL with $T_{0}=$ $270 \mathrm{MeV}$ is quite a bit higher than the LQCD prediction [45-48]. Following [49], we adopt $T_{0}=195 \mathrm{MeV}$ here, which can lead to a lower $T_{c}$.

The NJL part of PNJL has six parameters and a typical parameter set obtained in [50,51] is listed in Table II. These parameters are determined by the empirical values of $\eta^{\prime}$ and $\pi$ meson masses, the $\pi$ decay constant $f_{\pi}$, and the quark condensates at vacuum. To qualitatively investigate the sensitivity of the RW transition on the $\mathbb{Z}_{3}$ symmetry breaking patterns, we take the current quark masses as free parameters in this study, while keeping $G_{S}, G_{D}$, and $\Lambda$ unchanged.

\section{NUMERICAL RESULTS}

In this section, we show numerical results of PNJL with the imaginary chemical potentials $\left(\mu_{u}, \mu_{d}, \mu_{s}\right) / i T=$ $(\theta-2 C \pi / 3, \theta, \theta+2 C \pi / 3)$, where $0 \leq C \leq 1$. We study the RW and deconfinement transitions under conditions (i)-(v), respectively. We concentrate on how these transitions depend on the pattern of center symmetry breaking.

At high temperature, the thermodynamical potential of $\mathbb{Z}_{3}$-PNJL has three degenerate local minima at $\phi=0$ and $\pm 2 \pi / 3$, which are the three $\mathbb{Z}_{3}$ sectors. Correspondingly, the thermodynamic potential of PNJL may have three nondegenerate solutions [namely, $\Omega_{\phi}(\phi=0, \pm 2 \pi / 3)$ ], and the ground state $\Omega_{g s}$ is determined by the absolute minimum of the three.

Without loss of generality, we take a fixed high temperature $T=250 \mathrm{MeV}$ to do the calculations at which the RW transition always happens in this model.

\section{A. Center symmetry breaking pattern (i): $N_{f}=3$ with varied $C \neq 1$}

We first perform the calculation in $\mathbb{Z}_{3}$-PNJL with the small common quark mass $5.5 \mathrm{MeV}$. Figure 1 shows the thermodynamical potential $\Omega$ as the function of $\theta$ for two different temperatures. We confirm that the ground state has a threefold degeneracy at high temperature, and there is no degeneracy at low temperature. The high- $T$ degeneracy indicates the spontaneous center symmetry breaking, which 

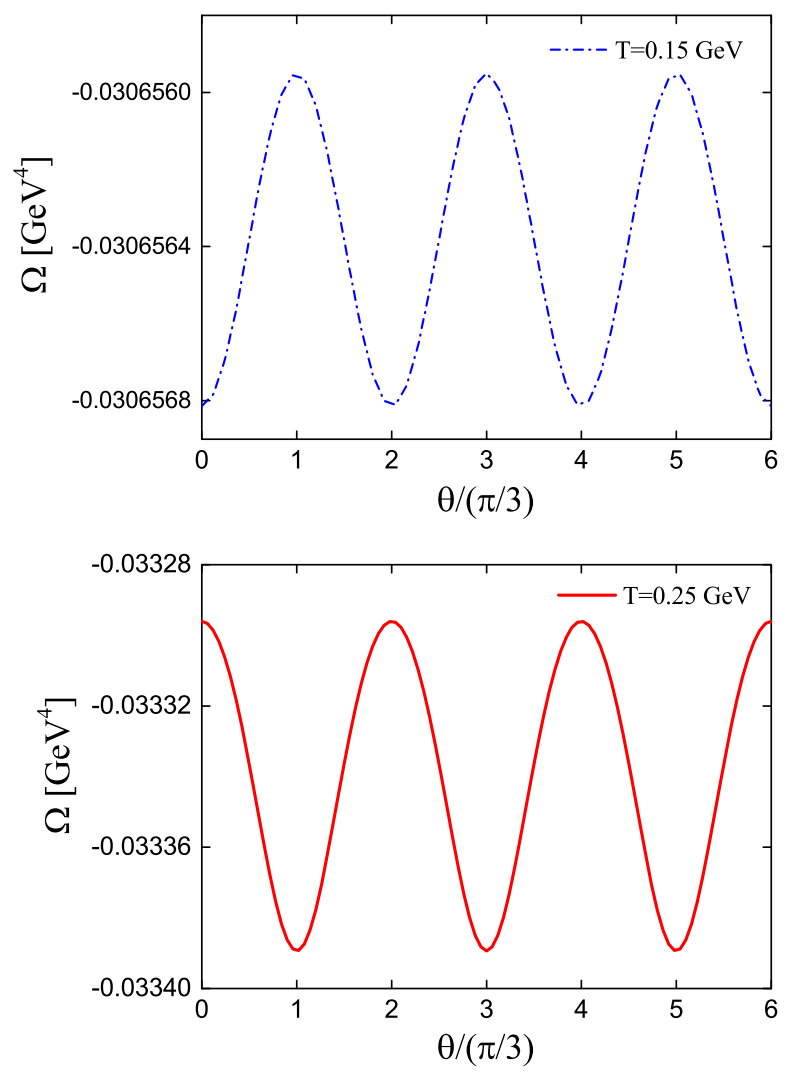

FIG. 1. Thermodynamic potential $\Omega$ as the function of $\theta$ for $T=150 \mathrm{MeV}$ (upper) and $T=250 \mathrm{MeV}$ (lower) in $\mathbb{Z}_{3}$-PNJL with $m_{u}=m_{d}=m_{s}=5.5 \mathrm{MeV}$ and $C=1$.

rules out the RW transition. Numerical calculation indicates that the critical temperature $T_{c} \approx 195 \mathrm{MeV}$, which is almost independent of $\theta$ [28]. We see that the RW periodicity always holds, even though the $\theta$ dependence of $\Omega$ at low- $T\left(<T_{c}\right)$ is quite a bit weaker than that at high- $T$ ( $>T_{c}$ ). The upper panel displays the fact that $\Omega(\theta)$ peaks at $\theta=(2 k+1) \pi / 3$ for $T=150 \mathrm{MeV}$, but the lower panel shows it peaks at $\theta=2 \mathrm{k} \pi / 3$ for $T=250 \mathrm{MeV}$. This implies that the $T$-driven first-order transition related to center symmetry corresponds to the shift of the shape of $\Omega(\theta)$. This is a nontrivial result in the center-symmetric theory with fermions.

Figure 2 shows $\Omega_{\phi}(\theta)$ at $T=250 \mathrm{MeV}$ for $N_{f}=3$ with the same common quark mass as in Fig. 1 , but $C \neq 1$, which corresponds to center symmetry breaking pattern (i). We see the shifts between three $\mathbb{Z}_{3}$ sectors appear in the $\theta-\Omega$ plane and the cusps of $\Omega$ emerge at $\theta=\theta_{\mathrm{RW}}=$ $(2 k+1) \pi / 3$. Note that the angle $\theta_{\mathrm{RW}}$ is consistent with the traditional one in QCD with $C=0$. Figure 2 displays that each $\Omega_{\phi}(\theta)$ has the period $2 \pi$, which is continuous (discontinuous) when center symmetry is weakly (strongly) broken for $C$ near one (zero). Figure 2(d) shows that the solution $\Omega_{0}(\theta)$ for $C=0.3$ vanishes in the region $0.6 \pi<$ $\theta<1.4 \pi$, which is similar to that of the standard RW transition obtained in the two-flavor PNJL [22]. We notice that the PNJL correctly reproduces the relation $\Omega_{g s}(\theta)=$ $\Omega_{g s}(-\theta)$ required by pattern (i). So the RW transitions shown in Fig. 2 still reflect the spontaneous breaking of $\mathbb{Z}_{2}$ symmetry and the density $\partial \Omega / \partial(i \theta)$ is the order parameter.

As expected, Fig. 2 shows that the RW cusps become sharper when $C$ declines from one to zero. The RW transition getting stronger with center symmetry breaking is demonstrated more clearly in the left panel of Fig. 3. In contrast, the deconfinement transition evaluated by the Polyakov loop becomes weaker with the decrease of $C$. This is shown in the right panel of Fig. 3, where the $\theta-T$ phase diagrams for three different $C^{\prime} s$ are plotted (the solid line denotes the first-order transition). In this panel, the vertical lines represent the RW transitions and the other lines represent the deconfinement transitions. We see that, for $C=0.8$, the whole line of deconfinement is first order, but for $C=0.5$ only the short line near the RW end point keeps first order. When $C$ approaches zero, the first-order line of deconfinement further shrinks toward the RW line but does not vanish at $C=0$. So all the RW end points for pattern (i) with a small quark mass are triple points in this model. ${ }^{1}$ Note that the triple point may change into a critical end point if the common quark mass is large enough. In this case, there should exist a critical value $C_{c}$ below which the RW end point is second order.

\section{B. Center symmetry breaking pattern (ii): $N_{f}=2+1$ with $C=1$}

This subsection gives the numerical results for pattern (ii) of $N_{f}=2+1$ and $C=1$, where the center symmetry of $\mathbb{Z}_{3}$-QCD is broken by the mass difference between two degenerate light flavors $(u$ and $d)$ and a heavy one $(s)$.

Figure 4 presents the thermodynamic potential $\Omega_{\phi}$ as the function of $\theta$ at $T=250 \mathrm{MeV}$ for two different $m_{s}$ 's, where $m_{l(u, d)}=5.5 \mathrm{MeV}$. In the range $0 \leq \theta<2 \pi$, each $\Omega_{\phi}$ has three local minimums for $m_{s}=10 \mathrm{MeV}$, but only one for $m_{s}=140.7 \mathrm{MeV}$; the shifts between three $\mathbb{Z}_{3}$ sectors appear in both cases. Similar to pattern (i), the relation $\Omega(\theta)=\Omega(-\theta)$ is also reproduced correctly in PNJL. Different from pattern (i), the RW cusps occur at $\theta=2 k \pi / 3$ rather than $(2 k+1) \pi / 3$.

Note that $\theta_{\mathrm{RW}}=2 k \pi / 3$ can be explained using the previous study [23], in which the RW transitions at finite imaginal baryon and isospin chemical potentials, namely, $\mu_{q(I)}=i T \theta_{q(I)}$, are investigated in an $N_{f}=2$ PNJL. The prediction of [23] is that (a) the RW transition emerges at $\theta_{q}=0 \bmod 2 \pi / 3$ when $-\pi / 2-\delta(T)<\theta_{I}<\pi / 2+\delta(T),{ }^{2}$ and (b) it does at $\theta_{q}=\pi / 3 \bmod 2 \pi / 3$ when $\pi / 2-$

\footnotetext{
${ }^{1}$ This is different from the current lattice predictions in that the physical RW end point may be second order. Note that, in PNJL with $C=0$, the nature of the RW end point depends on the Polyakov-loop potential.

${ }^{2}$ Here $\delta(T)=0.00016 \times(T-250)$.
} 

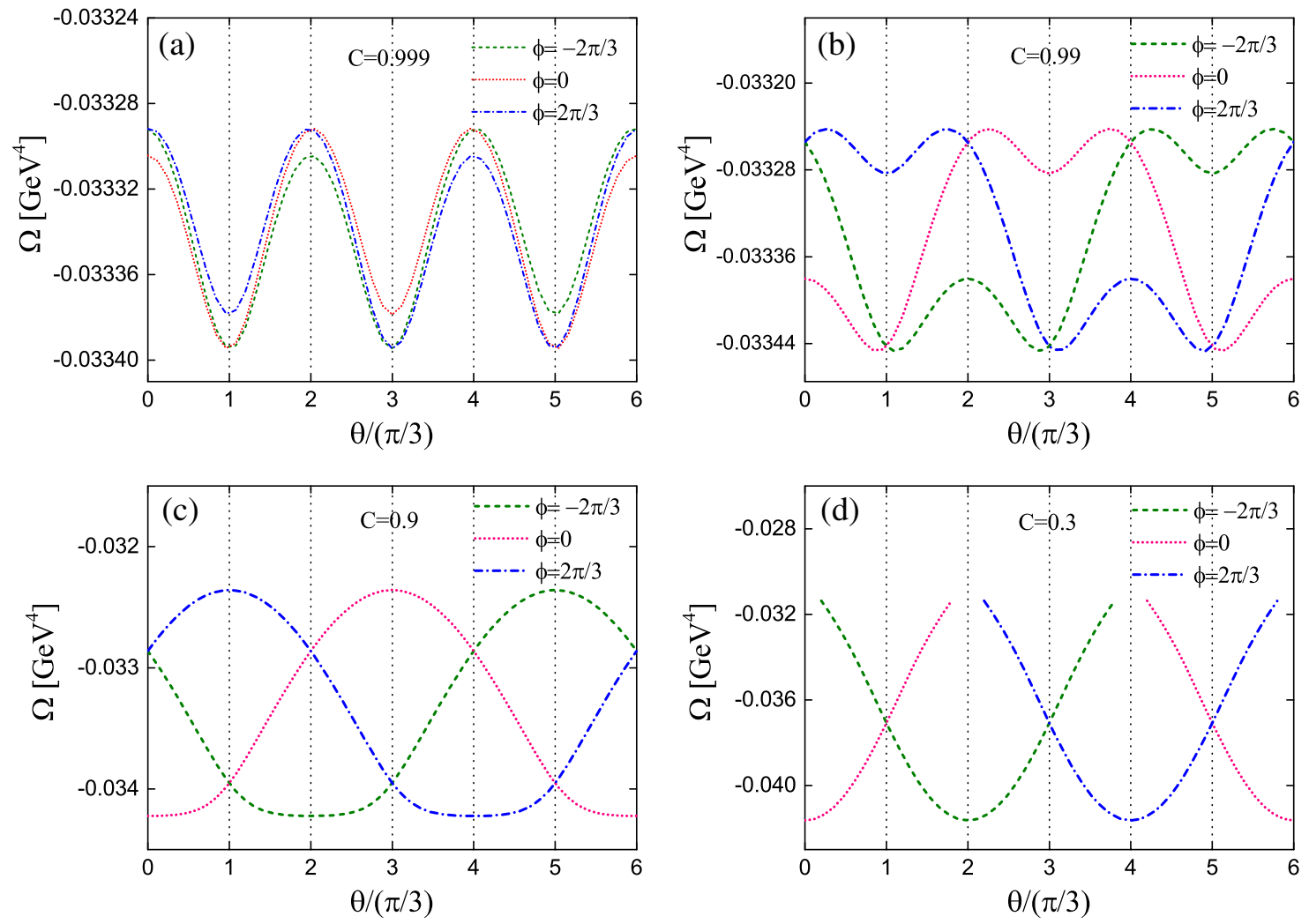

FIG. 2. Thermodynamic potentials of the $\mathbb{Z}_{3}$ sectors as the functions of $\theta$ at $T=250 \mathrm{MeV}$ in PNJL for $N_{f}=3$ with fixed $m_{u}=$ $m_{d}=m_{s}=5.5 \mathrm{MeV}$ and varied $C \neq 1$. In the calculation, we set (a) $C=0.999$, (b) $C=0.99$, (c) $C=0.9$, and (d) $C=0.3$, respectively.
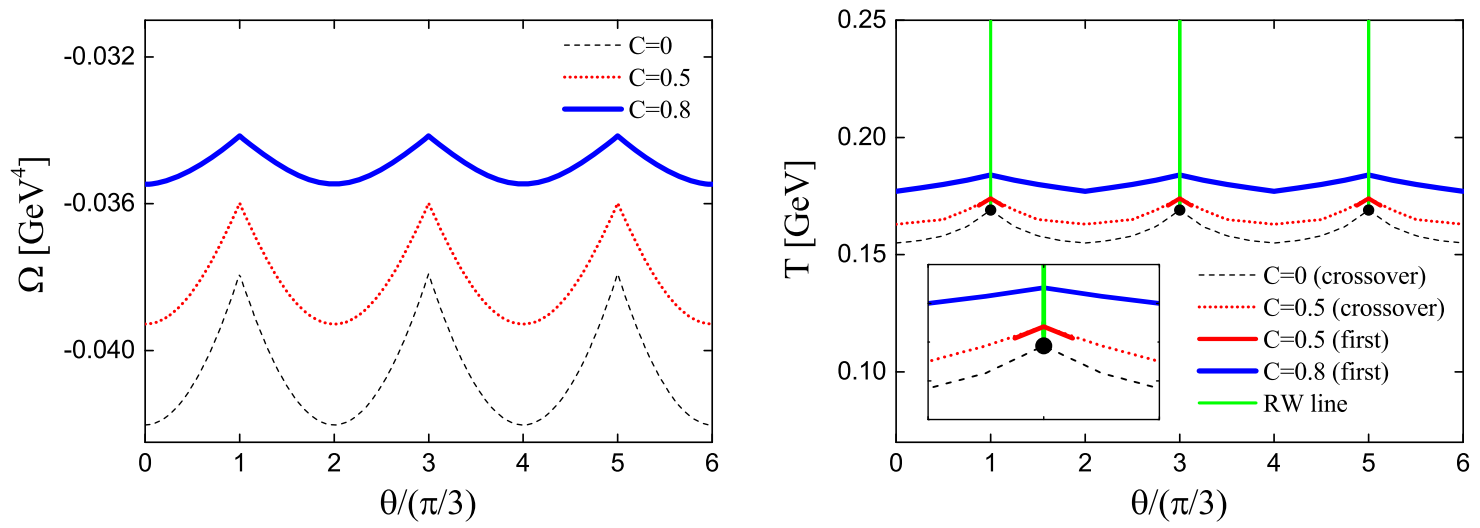

FIG. 3. Thermodynamic potential $\Omega$ as the function of $\theta$ at $T=250 \mathrm{MeV}$ (left) and the $\theta-T$ phase diagram (right) for $m_{u}=$ $m_{d}=m_{s}=5.5 \mathrm{MeV}$ with varied $C \neq 1$. The black spots on the right mean the RW end points at $C=0$ are still triple points.

$\delta(T)<\theta_{I}<3 \pi / 2+\delta(T)$. In our case, with $N_{f}=2+1$ and $C=1, \theta_{q}$ and $\theta_{I}$ associated with two light flavors are $((\theta-2 \pi / 3)+\theta) / 2=\theta-\pi / 3$ and $((\theta-2 \pi / 3)-\theta) /$ $2=-\pi / 3$, respectively. According to (a) (ignoring the heavy flavor for the moment), $\theta_{I}=-\pi / 3$ belongs to the range $(-\pi / 2-\delta(T), \pi / 2+\delta(T))$, and thus the RW transition appears at $\theta_{q}=\theta_{\mathrm{RW}}-\pi / 3=\pi / 3 \bmod 2 \pi / 3$. Namely, $\theta_{\mathrm{RW}}=2 k \pi / 3$. On the other hand, if we adopt $\left(\mu_{u}, \mu_{d}, \mu_{s}\right) / i T=(\theta-2 \pi / 3, \theta+2 \pi / 3, \theta)$, the corresponding $\theta_{q}$ and $\theta_{I}$ are $\theta$ and $-2 \pi / 3$, respectively. In this case,
$\theta_{I}+2 \pi=4 \pi / 3$ belongs to the range $(\pi / 2-\delta(T), 3 \pi /$ $2+\delta(T))$, and thus the RW transition occurs at $\theta_{q}=$ $\theta_{\mathrm{RW}}=0 \bmod 2 \pi / 3$ according to (b). So we still obtain $\theta_{\mathrm{RW}}=2 k \pi / 3$.

The consistency between our result and that in [23] implies that the RW angle for $N_{f}=2+1$ with $C=1$ is mainly determined by the two degenerate light flavors. Actually, we will show later that $\theta_{\mathrm{RW}}$ is still $(2 k+1) \pi / 3$ for $N_{f}=1+2$ with $C=1$, in which there is only one light flavor. 

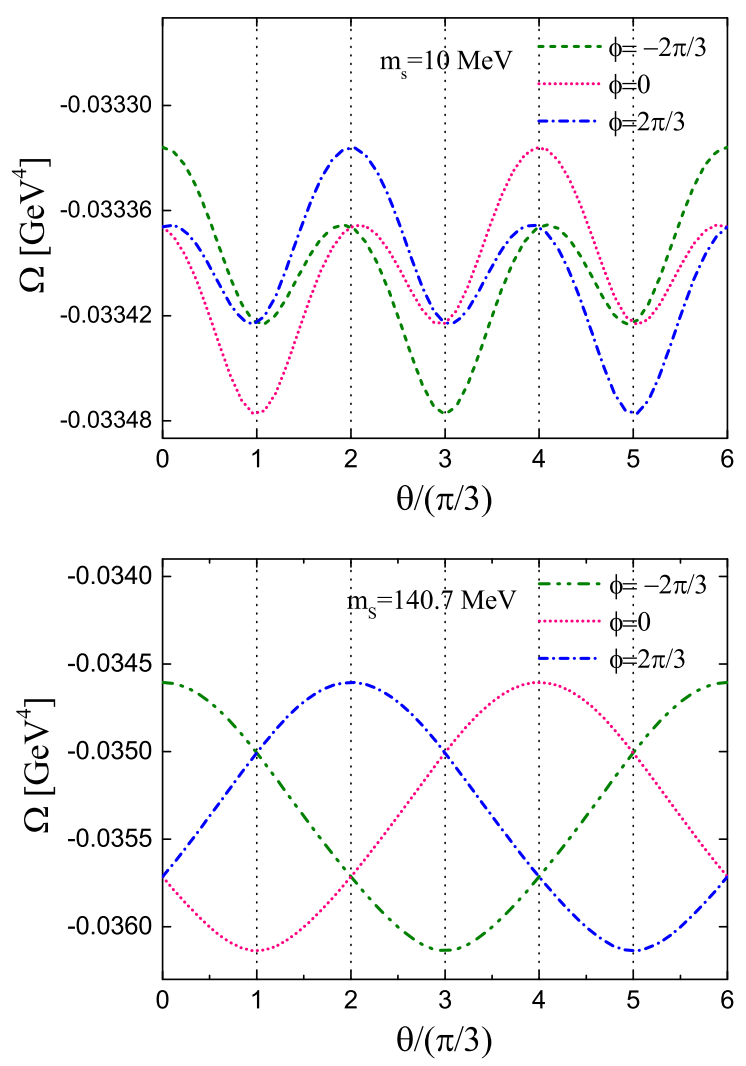

FIG. 4. Thermodynamic potentials of the $\mathbb{Z}_{3}$ sectors as the functions of $\theta$ at $T=250 \mathrm{MeV}$ with $C=1$ for $m_{s}=10 \mathrm{MeV}$ (upper) and $m_{s}=140.7 \mathrm{MeV}$ (lower). The masses of two light flavors are fixed as $m_{u}=m_{d}=5.5 \mathrm{MeV}$. The RW transitions appear at $\theta=2 k \pi / 3$.

Figure 5 displays the $\theta$ dependence of the quark number density $n_{I}=\operatorname{Im}\left(n_{q}\right)$ for $m_{l(u, d)}=5.5$ and $m_{s}=140.7 \mathrm{MeV}$. In pattern (ii), $n_{I}(\theta)$ is $\theta$ odd and thus the order parameter for $\mathbb{Z}_{2}$ symmetry. We see that it is continuous for $T=150 \mathrm{MeV}$ but discontinuous at $\theta=2 k \pi / 3$ for $T=250 \mathrm{MeV}$.

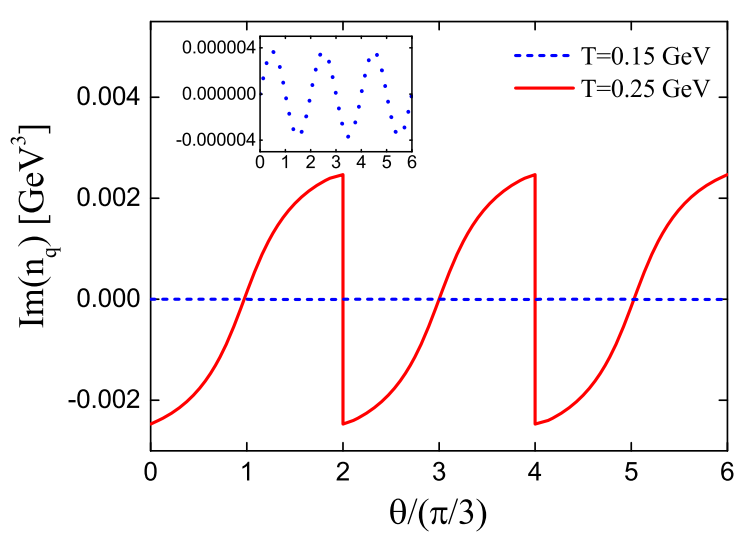

FIG. 5. The quark number density $\operatorname{Im}\left(n_{q}\right)$ as a function of $\theta$ for $C=1$ at $T=150 \mathrm{MeV}$ (dotted line) and $T=250 \mathrm{MeV}$ (solid line). The quark masses are same as that in the lower part of Fig. 4.

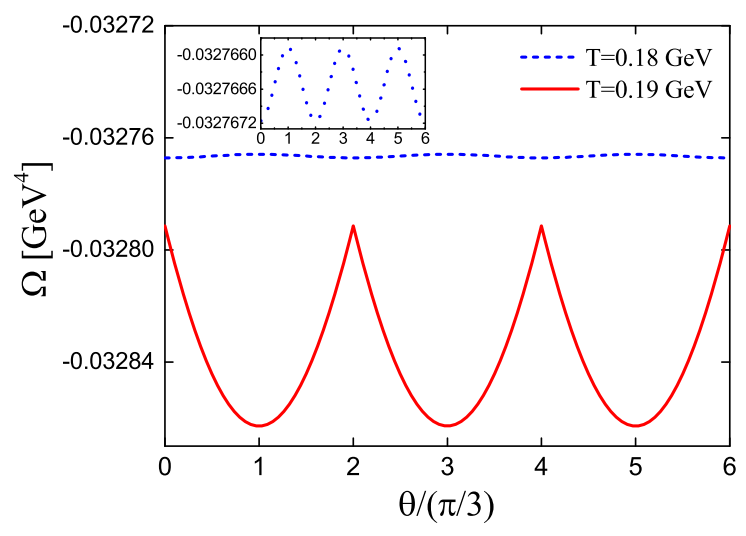

FIG. 6. Thermodynamic potential $\Omega$ as a function of $\theta$ for $C=$ 1 at $T=180 \mathrm{MeV}$ (dotted line) and $T=190 \mathrm{MeV}$ (solid line). The quark masses are same as that in the lower part of Fig. 4.

In Fig. 6, we compare the thermodynamic potentials for temperatures below and above $T_{\mathrm{RW}}$. For $T=180 \mathrm{MeV}$ $\left(<T_{\mathrm{RW}}\right), \Omega$ is weakly dependent on $\theta$, of which peaks and troughs are located at $\theta=(2 k+1) \pi / 3$ and $2 k \pi / 3$, respectively; but for $T=190 \mathrm{MeV}$ ( $>T_{\mathrm{RW}}$ ), it depends on $\theta$ obviously and the positions of peak and trough are exchanged. Note that the peak and trough locations of $\Omega$ at low and high temperature in Fig. 6 are all the same as that of $\mathbb{Z}_{3}$-PNJL shown in Fig. 1. This indicates that the center
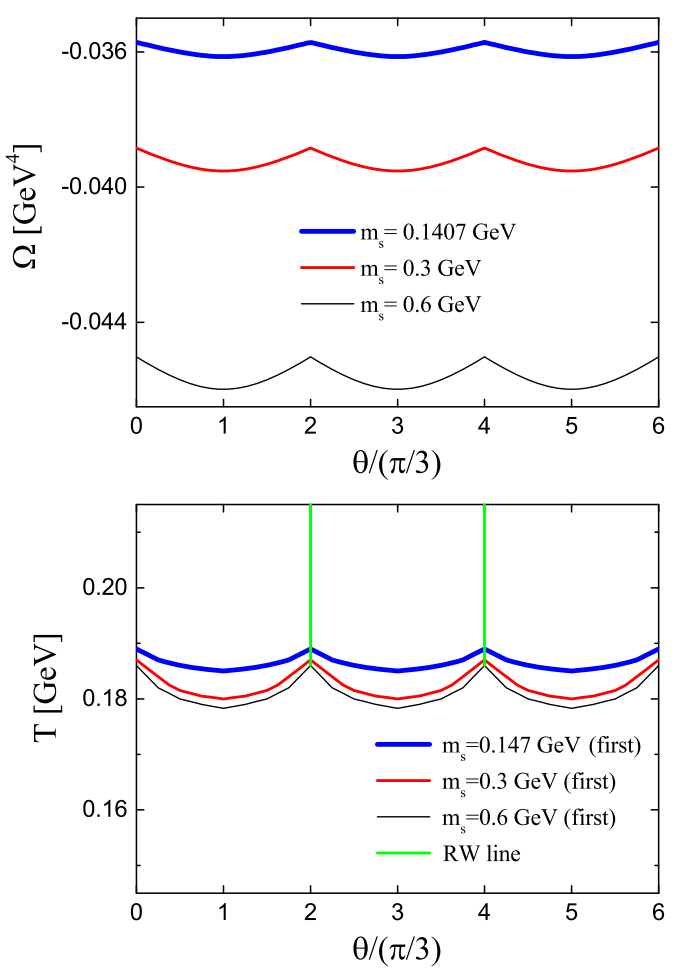

FIG. 7. (Upper) $\theta$ dependences of the thermodynamic potential $\Omega$ at $T=250 \mathrm{MeV}$ for $C=1$ and $m_{u}=m_{d}=5.5 \mathrm{MeV}$ with different strange quark masses. (Lower) $\theta-T$ phase diagrams under the same conditions. 


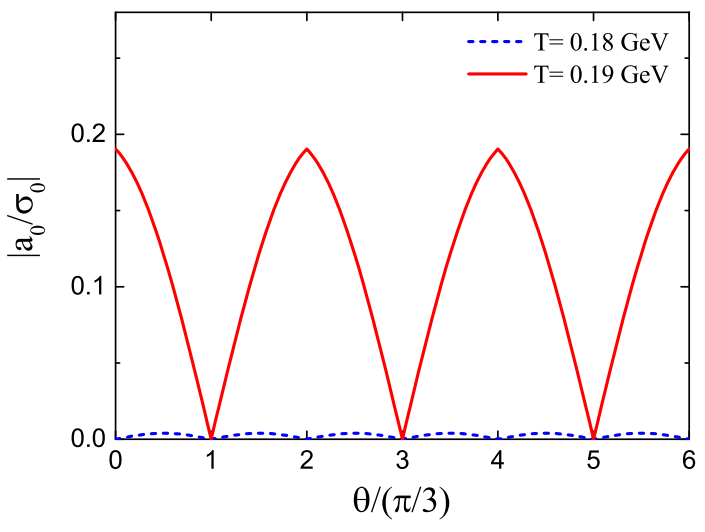

FIG. 8. The isovector condensate $a_{0}$ as a function of $\theta$ for $C=$ 1 at $T=180 \mathrm{MeV}$ (dotted line) and $190 \mathrm{MeV}$ (solid line). The quark masses are same as that in the lower part of Fig. 4.

symmetry is broken weakly in pattern (ii) with the physical quark masses.

The upper panel of Fig. 7 presents thermodynamic potentials as functions of $\theta$ at $T=250 \mathrm{MeV}$ for different larger $m_{s}$ 's $\left(m_{s}=140.7,300,600 \mathrm{MeV}\right)$ with fixed $m_{l}=5.5 \mathrm{MeV}$. As expected, the RW cusps become
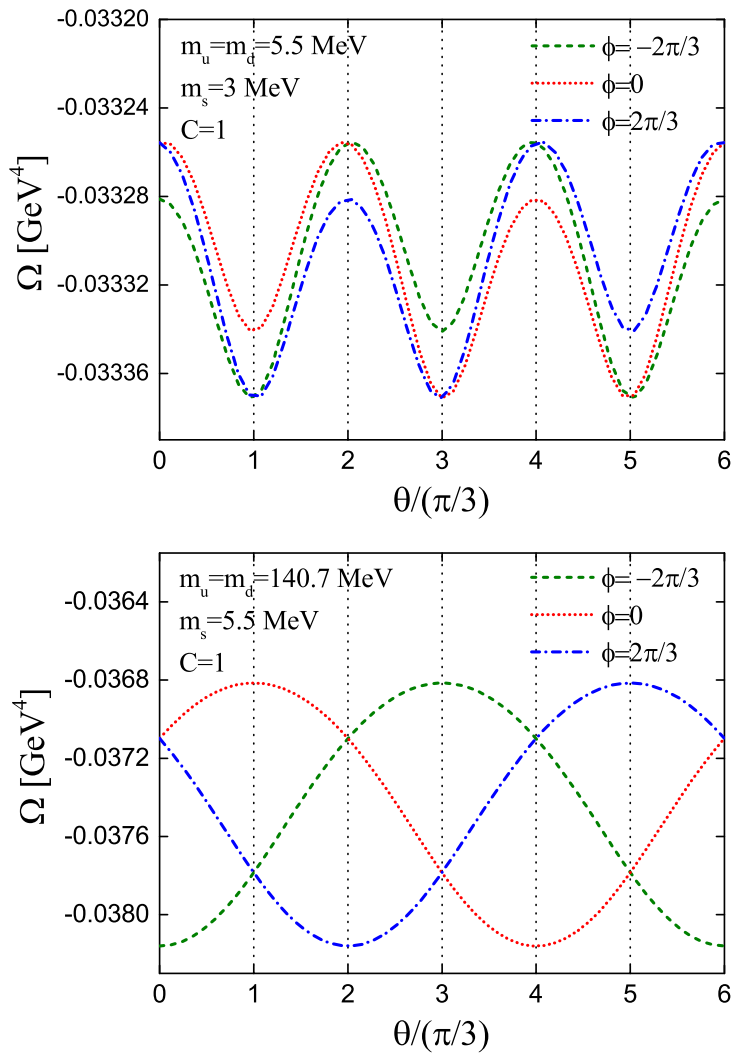

FIG. 9. Thermodynamic potentials of the $\mathbb{Z}_{3}$ sectors as the functions of $\theta$ at $T=250 \mathrm{MeV}$ for $N_{f}=1+2$ with $C=1$. (Upper) Corresponds to the case with $m_{u}=m_{d}=5.5$ and $m_{s}=3 \mathrm{MeV}$. (Lower) Corresponds to $m_{u}=m_{d}=140.7$ and $m_{s}=5.5 \mathrm{MeV}$. The RW transitions appear at $\theta=(2 k+1) \pi / 3$. sharper with $m_{s}$, but the change is milder (we only consider $m_{s}<\Lambda$ due to the limitation of PNJL). The lower panel of Fig. 7 displays the $\theta-T$ phase diagrams under the same conditions. The deconfinement transitions for those $m_{s}$ 's are all first order, and thus the RW end points are triple points. This suggests that the center symmetry is broken less severely by the mass differences considered here. Similar to Fig. 3, the lower panel shows that the higher the degree of center symmetry breaking, the lower the $T_{\mathrm{RW}}$. Another common feature of Figs. 3 and 7 is that $T_{\mathrm{RW}}$ is the highest critical temperature of deconfinement for a fixed $C \neq 1$ (former) or $m_{s}$ (later). This implies that the RW transition has a significant impact on the deconfinement transition in both symmetry breaking patterns.

In Fig. 8, we show the isovector condensate $a_{0}=$ $\langle\bar{u} u-\bar{d} d\rangle$ as a function of $\theta$ for $C=1$ with the physical quark masses. Here $a_{0}$ is normalized by $\sigma_{0} \equiv \sigma(T=0$, $\left.\mu_{f}=0\right)$, where $\sigma \equiv\left(\sigma_{u}+\sigma_{d}+\sigma_{s}\right) / 3$. For $T=180 \mathrm{MeV}$, the $a_{0} \sim 0$ apart from $\theta=k \pi / 3$ where $a_{0}=0$. This can be considered as a remanent of the $N_{f}=3$ case, where $a_{0}=0$ at low $T$ [28]. The approximate flavor symmetry at low $T$ is attributed to the color confinement where $\Phi \sim 0$. When $\theta=k \pi / 3$, the charge conjugate symmetry preserves the flavor symmetry between $u$ and $d$. Actually, under the $\mathcal{C}$
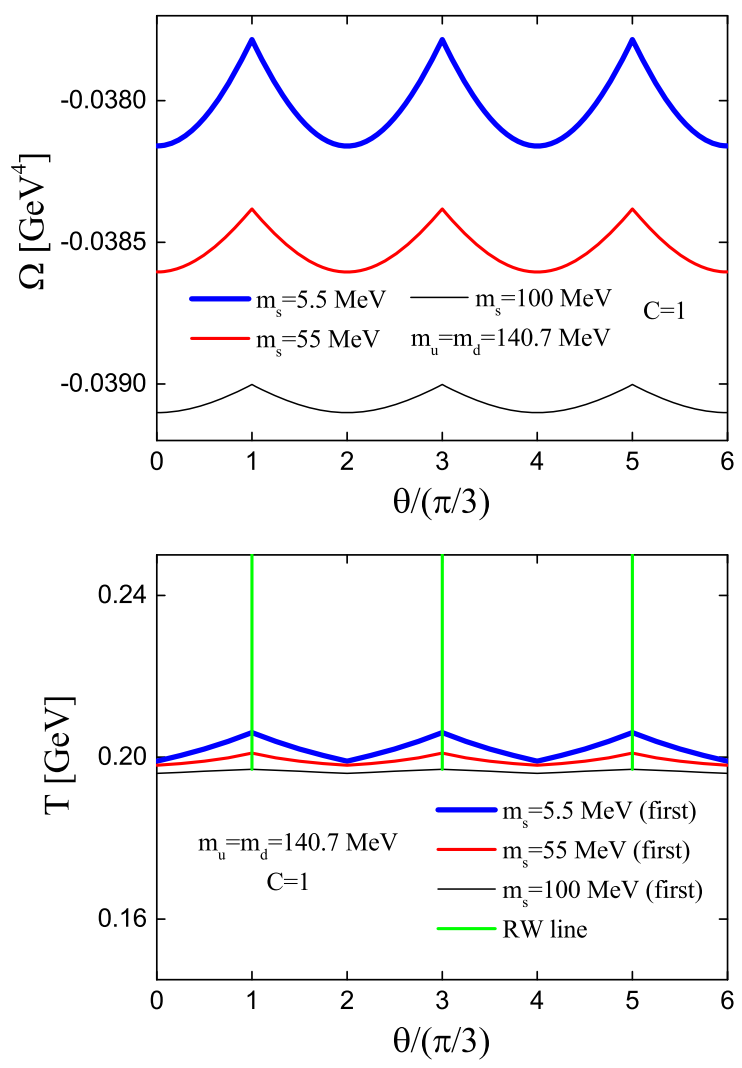

FIG. 10. (Upper) $\theta$ dependences of the thermodynamic potential $\Omega$ at $T=250 \mathrm{MeV}$ for different $m_{s}$ 's and fixed $C=1$, where $m_{u}=m_{d}=140.7 \mathrm{MeV}$. (Lower) The $\theta-T$ phase diagrams under the same conditions. 

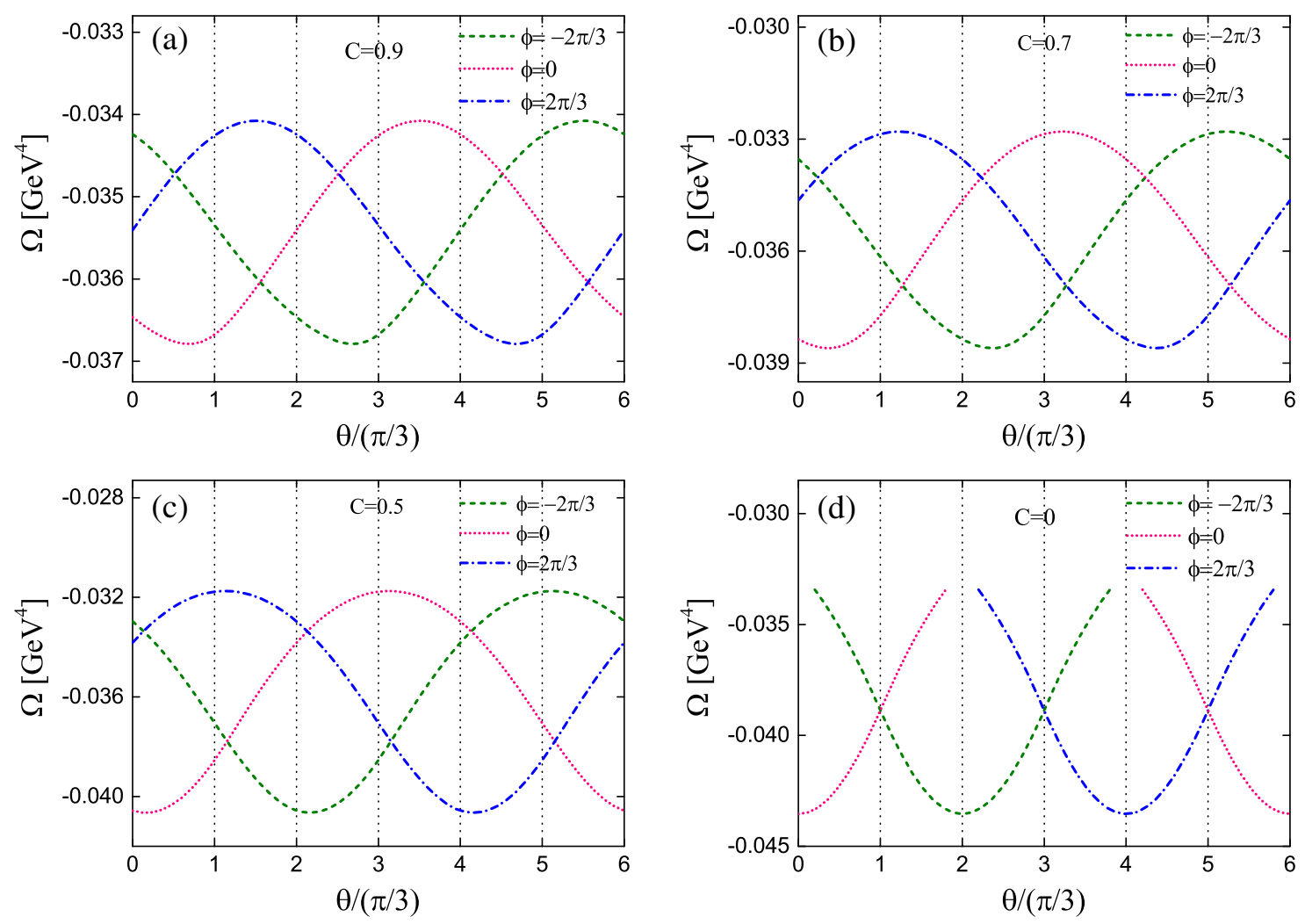

FIG. 11. Thermodynamic potentials of the $\mathbb{Z}_{3}$ sectors for different $C$ at $T=250 \mathrm{MeV}$ in the case of $N_{f}=2+1$ with $m_{u}=m_{d}=5.5$ and $m_{s}=140.7 \mathrm{MeV}$. The RW transition point moves from $2 k \pi / 3$ to $(2 k-1) \pi / 3$ when $C$ changes from one to zero. In the calculation, we set (a) $C=0.9$, (b) $C=0.7$, (c ) $C=0.5$, and (d) $C=0$, respectively.

transformation $\Phi \leftrightarrow \Phi^{*}$, the thermodynamic potential with $\theta=0$

$$
\begin{aligned}
& \Omega(-2 \pi / 3,0,2 \pi / 3) \stackrel{\mathcal{C}}{\longrightarrow} \Omega(2 \pi / 3,0,-2 \pi / 3) \stackrel{-2 \pi / 3}{=} \\
& \Omega(0,-2 \pi / 3,-4 \pi / 3) \frac{-4 \pi / 3 \rightarrow 2 \pi / 3}{u \leftrightarrow d} \Omega(-2 \pi / 3,0,2 \pi / 3),
\end{aligned}
$$

and that with $\theta=\pi / 3$

$$
\begin{aligned}
& \Omega(-\pi / 3, \pi / 3, \pi) \stackrel{\mathcal{C}}{\rightarrow} \Omega(\pi / 3,-\pi / 3,-\pi) \\
& \frac{-\pi \rightarrow \pi}{u \leftrightarrow d} \Omega(-\pi / 3, \pi / 3, \pi),
\end{aligned}
$$

where $u \leftrightarrow d$ stands for the relabeling of $u$ and $d$. For $T=190 \mathrm{MeV}$, the two-flavor symmetry is broken at $\theta=$ $2 k \pi / 3$ due to the RW transition $[22,28]$.

\section{Center symmetry breaking pattern (iii): $N_{f}=1+2$ with $C=1$}

The numerical results for $N_{f}=1+2$ and $C=1$ or pattern (iii) are given in this subsection. The center symmetry is broken by the mass difference between the light flavor and two degenerate heavy ones. Note that $s$ refers to the only light flavor here.
Figure 9 shows $\mathbb{Z}_{3}$ sectors of $\Omega$ as functions of $\theta$ at $T=250 \mathrm{MeV}$ for two cases with $m_{u(d)}=5.5$ and $m_{s}=$ $3 \mathrm{MeV}$ (upper panel) and $m_{u(d)}=140.7$ and $m_{s}=5.5 \mathrm{MeV}$ (lower panel). Different from pattern (ii), both panels display that RW transitions occur at $\theta=(2 k+1) \pi / 3$. This difference can be understood in the following way. In Fig. 9, the physical thermodynamic potentials in intervals $-\frac{\pi}{3}<\theta<\frac{\pi}{3}, \frac{\pi}{3}<\theta<\pi$, and $\pi<\theta<\frac{5 \pi}{3}$ are $\Omega_{\phi=-\frac{2 \pi}{3}}, \Omega_{\phi=\frac{2 \pi}{3}}$, and $\Omega_{\phi=0}$, respectively. Note that such an $\Omega_{\phi}$ order of the physical thermodynamic potential along the $\theta$ direction is same as that of a one-flavor system with $\mu=\mu_{s}=i\left(\theta+\frac{2 \pi}{3}\right) T^{3}$ This suggests that $\theta_{\mathrm{RW}}$ for $N_{f}=1+2$ and $C=1$ is mainly determined by the only light flavor. Such a conclusion also supports our argument that $\theta_{\mathrm{RW}}$ for $N_{f}=2+1$ and $C=1$ is mainly determined by the two degenerate light flavors.

The upper panel of Fig. 10 presents the $\theta$ dependences of $\Omega$ at $T=250 \mathrm{MeV}$ for fixed $m_{u(d)}=140.7 \mathrm{MeV}$ and varied $m_{s}\left(m_{s}<m_{u(d)}\right)$. As anticipated, the cusps of $\Omega$ become sharper with the decrease of $m_{s}$. The lower panel

\footnotetext{
${ }^{3}$ The $\Omega_{\phi}$ order of the thermodynamic potential for a one-flavor system with $\mu / i T=\theta$ is $\Omega_{\phi=0}, \Omega_{\phi=-\frac{2 \pi}{3}}$, and $\Omega_{\phi=\frac{2 \pi}{3}}$ for the $\theta$ intervals mentioned above [10]. When $\mu / i T=\theta+\frac{2 \pi}{3}$, the thermodynamic potential is shifted by $-\frac{2 \pi}{3}$ along the $\theta$ axis and thus the order becomes $\Omega_{\phi=-\frac{2 \pi}{3}}, \Omega_{\phi=\frac{2 \pi}{3}}$, and $\Omega_{\phi=0}$.
} 
shows the $\theta-T$ phase diagrams under the same conditions. Similar to Fig. 7, the deconfinement transitions are all first order, which suggests the center symmetry breaking due to mass deference is weak. We see that $T_{\mathrm{RW}}$ increases with the decrease of $m_{s}$. This means that the higher the degree of center symmetry breaking, the higher the $T_{\mathrm{RW}}$. This point is distinct with that shown in Figs. 3 and 7 and whether it is a model artifact is unclear.

\section{Center symmetry breaking pattern (iv): $N_{f}=2+1$ with varied $C \neq 1$}

Figure 11 shows the $\theta$ dependences of $\Omega_{\phi}$ at $T=$ $250 \mathrm{MeV}$ for different values of $C(C=0.9,0.7,0.5,0)$ with the physical quark masses. In these cases, the original center symmetry of $\mathbb{Z}_{3}$-QCD is explicitly broken by both the mass difference and $C \neq 1$.

Note that, for $N_{f}=2+1$, the relation $\Omega(\theta)=\Omega(-\theta)$, which is true for $C=0$ or 1 , does not hold when $C \in(0,1)$. Correspondingly, the angle $\theta_{\mathrm{RW}}$ for $0<C<$ 1 is located between $(2 k-1) \pi / 3$ and $2 k \pi / 3$, which moves toward $(2 k-1) \pi / 3(2 k \pi / 3)$ when $C \rightarrow 0(C \rightarrow 1)$, as demonstrated in Fig. 11. This figure clearly shows that the tip of the cusp becomes sharper with the decrease of $C$ and thus the standard RW transition [Fig. 11(d) represents the standard RW transition] is the strongest.

We do not plot the $\theta-T$ phase diagrams for this pattern with physical quark masses. The traditional RW end point in PNJL with physical quark masses is a triple point [26]. So we can expect that the phase diagrams for different values of $C$ are similar to Fig. 3 and the RW end points are triple ones, except $\theta_{\mathrm{RW}} \neq k \pi / 3$.

\section{E. Center symmetry breaking pattern (v): $N_{f}=1+1+1$ with $C=1$}

The $\theta$ dependences of $\Omega_{\phi}$ at $T=250 \mathrm{MeV}$ in pattern (v) are shown in Fig. 12, where $m_{u}=5.5, m_{d}=55$, and $m_{s}=140.7 \mathrm{MeV}$ are adopted. Similar to patterns (ii) and

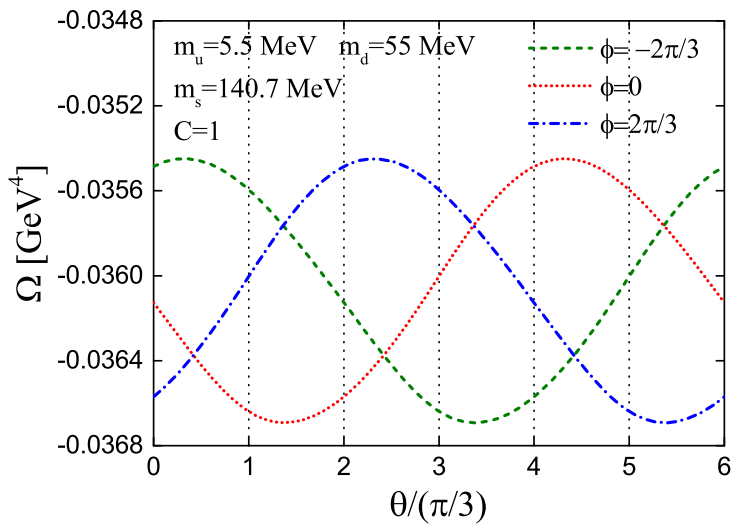

FIG. 12. Thermodynamic potentials of the $\mathbb{Z}_{3}$ sectors at $T=250 \mathrm{MeV}$ for $C=1$ in the case of $N_{f}=1+1+1$ with $m_{u}=5.5, m_{d}=55$, and $m_{s}=140.7 \mathrm{MeV}$. (iii), the original center symmetry of $\mathbb{Z}_{3}-\mathrm{QCD}$ is explicitly broken due to mass nondegeneracy.

Note that $\Omega(\theta) \neq \Omega(-\theta)$ in this pattern since different flavors have different masses. As a result, the RW transitions do not occur at $\theta=k \pi / 3$. Figure 12 shows that $\theta_{\mathrm{RW}}$ is in between $2 k \pi / 3$ and $(2 k+1) \pi / 3$, which is different from Fig. 11. Fixing $m_{u}$ and $m_{s}$ and keeping $m_{u}<m_{d}<m_{s}$, we verify that the RW point moves toward $2 k \pi / 3$ $[(2 k+1) \pi / 3]$ when $m_{d} \rightarrow m_{u}\left(m_{s}\right)$. This is easily understood since the condition $m_{u}=m_{d}<m_{s}\left(m_{u}<m_{d}=m_{s}\right)$ with $C=1$ corresponds to pattern (ii) [(iii)].

In pattern (v), how the RW transition depends on quark masses is complicated and the PNJL model is only suited to study the system with light quarks. Figure 12 shows that the strength of the RW transition is similar to cases of pattern (ii) with $m_{u(d)}=5.5$ and $m_{s}=140.7 \mathrm{MeV}$ and pattern (iii) with $m_{u(d)}=140.7$ and $m_{s}=5.5 \mathrm{MeV}$. This may suggest that in light flavor cases the RW transition due to mass nondegeneracy is quite a bit weaker than the traditional RW transition, and thus the center symmetry breaking is not so severe.

\section{DISCUSSION AND CONCLUSION}

In this paper, we use the three-flavor PNJL as a $\mathbb{Z}_{3}$-QCD model to investigate the nature of RW and deconfinement transitions by breaking the center symmetry in different patterns. The FTBCs are adopted, which correspond to the flavor-dependent imaginary chemical potentials $\left(\mu_{u}, \mu_{d}, \mu_{s}\right) / i T=(\theta-2 C \pi / 3, \theta, \theta+2 C \pi / 3)$. The center symmetry of $\mathbb{Z}_{3}$-QCD is explicitly broken when three flavors are mass nondegenerate and/or $C \neq 1$.

We first demonstrate that the thermodynamic potential $\Omega(\theta)$ for $N_{f}=3$ and $C=1$ peaks at $\theta=(2 k+1) \pi / 3$ and $2 k \pi / 3$ in low and high temperatures, respectively. Namely, the shift of the peak position of $\Omega(\theta)$ from $\theta=(2 k+1) \pi / 3$ to $\theta=2 \mathrm{k} \pi / 3$ with $T$ just corresponds to the true first-order deconfinement transition. There is no RW transition in this case because of the exact center symmetry.

For $N_{f}=3$ with $C \neq 1$, the RW transitions occur at $\theta=(2 k+1) \pi / 3$ when $T>T_{\mathrm{RW}}$. The transition strength becomes stronger when $C$ decreases from one and the strongest corresponds to the traditional RW transition with $C=0$. We verify that the RW end point is always a triple point in the light flavor case with $m_{u}=m_{d}=$ $m_{s}=5.5 \mathrm{MeV}$. The corresponding first-order deconfinement transition line in the $\theta-T$ plane becomes shorter when $C$ approaches zero. For $C$ near zero, the first-order deconfinement transition only appears at a very small region around the RW end point.

For $N_{f}=2+1$ with $C=1$, the RW transitions appear at $\theta=2 k \pi / 3$ rather than $\theta=(2 k+1) \pi / 3$. We argue that the angle $\theta_{\mathrm{RW}}$ in this case is mainly determined by the two mass-degenerate light flavors, which is supported by the previous study for the two-flavor system at nonzero imaginary baryon and isospin chemical potentials [23]. 
The only heavier flavor affects the $T_{\mathrm{RW}}$ and RW strength directly. For $m_{u}=m_{d}=5.5 \mathrm{MeV}$, it is found that the tips of RW cusps become sharper with $m_{s}\left(m_{s}>m_{u}\right)$; moreover, the RW end points are always triple points and only the first-order deconfinement transition appears in the $\theta-T$ plane.

In contrast, the RW transitions for $N_{f}=1+2$ and $C=1$ still appear at $\theta=(2 k+1) \pi / 3$. This is because the $\theta_{\mathrm{RW}}$ in this pattern is determined by the only light flavor, rather than the two degenerate heavier ones, which is consistent with the argument mentioned above. Similarly, the flavor mass mismatch impacts the $T_{\mathrm{RW}}$ and RW strength significantly. For $m_{u}=m_{d}=140.7 \mathrm{MeV}$ and $m_{s}<m_{u}$, it is found that, with the decrease of $m_{s}$, the RW transition gets stronger but the $T_{\mathrm{RW}}$ becomes higher. The latter is unusual in comparison with the aforementioned two cases and the reason is unclear. Similar to the pattern of $N_{f}=2+1$ and $C=1$, the deconfinement transition is always first order.

In the above three patterns, the relation $\Omega(\theta)=\Omega(-\theta)$ holds and the $\theta_{\mathrm{RW}}$ 's are integral multiples of $\pi / 3$. In general, $\Omega(\theta)$ is not $\theta$ even and the $\theta_{\mathrm{RW}}$ 's can be other values. For $N_{f}=2+1$ but $C \in(0,1)$, the $\theta_{\mathrm{RW}}$ is located in $((2 k-1) \pi / 3,2 k \pi / 3)$, which moves to $2 k \pi / 3((2 k-1) \times$ $\pi / 3$ ) when $C$ approaches one (zero). In this pattern, the RW strength is more sensitive to the deviation of $C$ from one rather than the mass difference. In contrast, for $N_{f}=$ $1+1+1$ with $C=1$, the $\theta_{\mathrm{RW}}$ is located in $(2 k \pi / 3$, $(2 k+1) \pi / 3)$, which moves toward $2 k \pi / 3((2 k+1) \pi / 3)$ when $N_{f}=1+1+1 \rightarrow N_{f}=2+1\left(N_{f}=1+2\right)$.

Our calculation suggests that the deconfinement transition always keeps first order for $C=1$ with or without the mass degeneracy in PNJL. This indicates that the center symmetry breaking caused purely by mass difference is too weak to lead to deconfinement crossover if the common difference of the $\mu_{f} / i T$ series is $2 \pi / 3$ in this model. In contrast, when $C$ deviates from one and below some critical value $C_{c}(\theta)$, the crossover for deconfinement occurs at $\theta$ far from $\theta_{\mathrm{RW}}$, which implies the strong center symmetry breaking. The first-order deconfinement transition line in the $\theta-T$ plane shrinks with the decrease of $C$ up to zero. Thus, the strongest deconfinement transition happens at $\theta_{\mathrm{RW}}$ and $C=0$.

The study gives predictions of how the RW and deconfinement transitions depend on the degree and manner of the center symmetry breaking related to $\mathbb{Z}_{3}$-QCD. These results may be illuminating to understand the relationship between $\mathbb{Z}_{3}$ symmetry, RW transition, and deconfinement transition at finite imaginary chemical potential and temperature regions where the LQCD simulations are available. The conclusions obtained here are mainly based on the effective model analysis, which should be checked by other methods. Moreover, the quark masses cannot be large enough in our calculation and it is unclear how the RW transition depends on the center symmetry breaking for the heavy quark system. Further study is necessary by employing the LQCD simulations or the perturbative weak coupling QCD by taking into account FTBCs.

\section{ACKNOWLEDGMENTS}

This work was supported by the National Natural Science Foundation of China (NSFC) (Grants No. 11875127, No. 11275069, No. 11605056).
[1] M. D’Elia, Nucl. Phys. A982, 99 (2019).

[2] J. B. Kogut and D. K. Sinclair, Phys. Rev. D 77, 114503 (2008).

[3] Z. Fodor and S. D. Katz, Phys. Lett. B 534, 87 (2002).

[4] Z. Fodor and S. D. Katz, J. High Energy Phys. 03 (2002) 014.

[5] C. R. Allton, S. Ejiri, S. J. Hands, O. Kaczmarek, F. Karsch, E. Laermann, C. Schmidt, and L. Scorzato, Phys. Rev. D 66, 074507 (2002).

[6] S. Ejiri, C. R. Allton, S. J. Hands, O. Kaczmarek, F. Karsch, E. Laermann, C. Schmidt, and L. Scorzato, Prog. Theor. Phys. Suppl. 153, 118 (2004).

[7] P. de Forcrand and O. Philipsen, Nucl. Phys. B642, 290 (2002).

[8] P. de Forcrand and O. Philipsen, Nucl. Phys. B673, 170 (2003).

[9] M. D’Elia, F. Di Renzo, and M. P. Lombardo, Phys. Rev. D 76, 114509 (2007).
[10] A. Roberge and N. Weiss, Nucl. Phys. B275, 734 (1986).

[11] M. D'Elia and F. Sanfilippo, Phys. Rev. D 80, 111501(R) (2009).

[12] P. de Forcrand and O. Philipsen, Phys. Rev. Lett. 105, 152001 (2010).

[13] C. Bonati, G. Cossu, M. D'Elia, and F. Sanfilippo, Phys. Rev. D 83, 054505 (2011).

[14] L.-K. Wu and X.-F. Meng, Phys. Rev. D 87, 094508 (2013).

[15] O. Philipsen and C. Pinke, Phys. Rev. D 89, 094504 (2014).

[16] L.-K. Wu and X.-F. Meng, Phys. Rev. D 90, 094506 (2014).

[17] C. Pinke and O. Philipsen, Proc. Sci., LATTICE2015 (2016) 149 [arXiv:1508.07725].

[18] C. Czaban, F. Cuteri, O. Philipsen, C. Pinke, and A. Sciarra, Phys. Rev. D 93, 054507 (2016).

[19] C. Bonati, M. D'Elia, M. Mariti, M. Mesiti, F. Negro, and F. Sanfilippo, Phys. Rev. D 93, 074504 (2016). 
[20] C. Bonati, E. Calore, M. D'Elia, M. Mesiti, F. Negro, F. Sanfilippo, S. F. Schifano, G. Silvi, and R. Tripiccione, Phys. Rev. D 99, 014502 (2019).

[21] Y. Sakai, K. Kashiwa, H. Kouno, and M. Yahiro, Phys. Rev. D 78, 036001 (2008).

[22] H. Kouno, Y. Sakai, K. Kashiwa, and M. Yahiro, J. Phys. G 36, 115010 (2009).

[23] Y. Sakai, H. Kouno, and M. Yahiro, J. Phys. G 37, 105007 (2010).

[24] K. Morita, V. Skokov, B. Friman, and K. Redlich, Phys. Rev. D 84, 076009 (2011).

[25] H. Kouno and T. Makiyama, J. Phys. G 40, 095003 (2013).

[26] J. Sugano, H. Kouno, and M. Yahiro, Phys. Rev. D 96, 014028 (2017).

[27] K. Kashiwa and A. Ohnishi, Phys. Rev. D 93, 116002 (2016); Phys. Lett. B 750, 282 (2015).

[28] H. Kouno, Y. Sakai, T. Makiyama, K. Tokunaga, T. Sasaki, and M. Yahiro, J. Phys. G 39, 085010 (2012).

[29] A. Cherman, S. Sen, M. Unsal, M. L. Wagman, and L. G. Yaffe, Phys. Rev. Lett. 119, 222001 (2017).

[30] Y. Sakai, H. Kouno, T. Sasaki, and M. Yahiro, Phys. Lett. B 718, 130 (2012).

[31] H. Kouno, T. Makiyama, T. Sasaki, Y. Sakai, and M. Yahiro, J. Phys. G 40, 095003 (2013).

[32] T. Iritani, E. Itou, and T. Misumi, J. High Energy Phys. 11 (2015) 159.

[33] H. Kouno, K. Kashiwa, J. Takahashi, T. Misumi, and M. Yahiro, Phys. Rev. D 93, 056009 (2016).

[34] Y. Liu, E. Shuryak, and I. Zahed, Phys. Rev. D 94, 105013 (2016).

[35] Y. Tanizaki, Y. Kikuchi, T. Misumi, and N. Sakai, Phys. Rev. D 97, 054012 (2018).
[36] W. j. Fu, Z. Zhang, and Y. x. Liu, Phys. Rev. D 77, 014006 (2008).

[37] K. Fukushima, Phys. Rev. D 77, 114028 (2008); 78, 039902 (E) (2008).

[38] Y. Sakai, K. Kashiwa, H. Kouno, and M. Yahiro, Phys. Rev. D 77, 051901(R) (2008).

[39] G. 't Hooft, Phys. Rev. Lett. 37, 8 (1976); Phys. Rev. D 14, 3432 (1976).

[40] M. Kobayashi and T. Maskawa, Prog. Theor. Phys. 44, 1422 (1970).

[41] M. Kobayashi, H. Kondo, and T. Maskawa, Prog. Theor. Phys. 45, 1955 (1971).

[42] S. Rößner, C. Ratti, and W. Weise, Phys. Rev. D 75, 034007 (2007).

[43] G. Boyd, J. Engels, F. Karsch, E. Laermann, C. Legeland, M. Lütgemeire, and B. Petersson, Nucl. Phys. B469, 419 (1996).

[44] O. Kaczmarek, F. Karsch, P. Petreczky, and F. Zantow, Phys. Lett. B 543, 41 (2002).

[45] E. Laermann and O. Philipsen, Annu. Rev. Nucl. Part. Sci. 53, 163 (2003).

[46] Z. Fodor and S. D. Katz, arXiv:0908.3341.

[47] S. Borsanyi, Z. Fodor, C. Hoelbling, S. D. Katz, S. Krieg, C. Ratti, and K. K. Szabó, J. High Energy Phys. 09 (2010) 073.

[48] A. Bazavov et al. (HotQCD Collaboration), Phys. Lett. B 795, 15 (2019).

[49] T. Sasaki, Y. Sakai, H. Kouno, and M. Yahiro, Phys. Rev. D 84, 091901(R) (2011).

[50] P. Rehberg, S. P. Klevansky, and J. Hüfner, Phys. Rev. C 53, 410 (1996).

[51] S. P. Klevansky, Rev. Mod. Phys. 64, 649 (1992). 